\title{
Imaging Pulmonary Blood Vessels and Ventilation-Perfusion Mismatch in COVID-19
}

\author{
Dnyanesh N. Tipre ${ }^{1}$, Michal Cidon ${ }^{2}$, and Rex A. Moats ${ }^{1}$ \\ ${ }^{1}$ Translational Biomedical Imaging Laboratory, Department of Radiology, Children's Hospital Los Angeles, The Saban Research \\ Institute, 4650 Sunset Blvd Rm 305, Los Angeles, CA, USA \\ ${ }^{2}$ Department of Rheumatology, Children's Hospital Los Angeles, Los Angeles, CA, USA 2022
}

\begin{abstract}
COVID-19 hypoxemic patients although sharing a same etiology (SARS-CoV-2 infection) present themselves quite differently from one another. Patients also respond differently to prescribed medicine and to prone Vs supine bed positions. A severe pulmonary ventilation-perfusion mismatch usually triggers moderate to severe COVID-19 cases. Imaging can aid the physician in assessing severity of COVID-19. Although useful for their portability X-ray and ultrasound serving on the frontline to evaluate lung parenchymal abnormalities are unable to provide information about pulmonary vasculature and blood flow redistribution which is a consequence of hypoxemia in COVID-19. Advanced imaging modalities such as computed tomography, single-photon emission tomography, and electrical impedance tomography use a sharp algorithm visualizing pulmonary ventilation-perfusion mismatch in the abnormal and in the apparently normal parenchyma. Imaging helps to access the severity of infection, lung performance, ventilation-perfusion mismatch, and informs strategies for medical treatment. This review summarizes the capacity of these imaging modalities to assess ventilation-perfusion mismatch in COVID-19. Despite having limitations, these modalities provide vital information on blood volume distribution, pulmonary embolism, pulmonary vasculature and are useful to assess severity of lung disease and effectiveness of treatment in COVID-19 patients.
\end{abstract}

Key words COVID-19 - CT angiography · HRCT - SPECT-CT · Electrical impedance tomography

\author{
Abbreviations \\ ${ }^{99 \mathrm{~m}}$ Tc-MAA Technetium ${ }^{99 \mathrm{~m}}$ Tc macro-aggregated \\ albumin \\ 99m Tc-DTPA Technetium \\ ${ }^{99 \mathrm{~m}}$ Tc-diethylen-tetraamino-pentaacetate \\ Technegas® Ultra-fine dispersion of Technetium \\ ${ }^{99 \mathrm{~m}}$ Tc-Carbon \\ ${ }^{81} \mathrm{Kr} \quad{ }^{81} \mathrm{~m}$ Krypton gas \\ ARDS Acute respiratory distress syndrome \\ BV Blood vessels
}

Correspondence to: Dnyanesh N. Tipre; e-mail: dnyanesht@ live.com
COVID-19 Coronavirus disease 2019

CTPA Conventional computed tomography pulmonary angiography

DECT Dual energy computed tomography

EIT Electrical impedance tomography

FRI Functional respiratory imaging

GGO Ground-glass opacities

HRCT High-resolution computed tomography

PEEP Positive end-expiratory pressure

SPECT Single photon emission tomography

U-HRCT Ultra high-resolution computed tomography VQ 


\section{Key points}

A severe pulmonary ventilation-perfusion mismatch triggers moderate to severe hypoxemia in COVID-19 cases.

Using a sharp algorithm, HRCT/U-HRCT, DECT, CTPA, SPECT, and EIT can visualize VQ mismatch, pulmonary embolism, pulmonary vasculature, and measure blood flow redistribution in the lungs.

Imaging modalities can aid the physician to access the severity of infection and lung performance, decide strategies for medical treatment, and evaluate response to treatment in patients.

\section{Introduction}

\section{Pulmonary Ventilation-Perfusion Mismatch in COVID-19}

Pulmonary ventilation is the process of air flowing into the lungs during inspiration (inhalation) and out of the lungs during expiration (exhalation). Gas exchange occurs in alveoli, and for effective gas exchange to occur, alveoli must be ventilated and perfused. Ventilation $(\mathrm{V})$ refers to the flow of air into and out of the alveoli, while perfusion $(\mathrm{Q})$ refers to the flow of blood to alveolar capillaries. These two variables, $\mathrm{V}$ and $\mathrm{Q}$, determine oxygen $\left(\mathrm{O}_{2}\right)$ and carbon dioxide $\left(\mathrm{CO}_{2}\right)$ levels in the blood. Normal $\mathrm{V}$ is $4 \mathrm{l} / \mathrm{min}$ of air and normal $\mathrm{Q}$ is $5 \mathrm{l} / \mathrm{min}$ of blood. So normal V/Q ratio is $4 / 5=0.8$ [1]. The actual values in the human lung vary depending on the position within the lung due to the gravitational effect. A
VQ mismatch is the term used when the ventilation and the perfusion at the alveolar site are not matched [2, 3]. A VQ mismatch is indicative of respiratory distress or failure in COVID-19 patients [4]. Extreme VQ mismatch is the result of an area with perfusion, but no ventilation $(\mathrm{V} / \mathrm{Q}=0)$ termed a "shunt" or an area with ventilation, but no perfusion $(\mathrm{V} / \mathrm{Q}=$ undefined) termed a "dead space" (Fig. 1a-c). In severe hypoxemic COVID-19 cases, hypoperfusion to the normal parenchyma due to vasoconstriction of pulmonary blood vessels results in poor gas exchange. The shifting of the blood pool from vasoconstricted small capillaries to the dilated blood vessels (redistribution) in ground-glass opacities (GGO) and consolidation results in shunting (Fig. 1d, e).

The fluid in alveoli triggers a pulmonary shunt and although perfused, the lungs remain unventilated. Intrapulmonary shunting causes hypoxemia (inadequate blood oxygen) in which the lungs become consolidated. Even breathing $100 \%$ oxygen does not result in fully oxygenated blood. Pulmonary embolism decreases perfusion relative to ventilation results in increased dead space. Dead zones usually can be corrected by supplying 100\% inspired oxygen. Even when a capillary is blocked because the pulsating nature of blood flow traveling forward and backward distributes between other capillaries that are exchanging gases efficiently $[5,6]$.

\section{Imaging Lung Manifestation and VQ Mismatch in COVID-19}

In COVID-19, severe hypoxemia is caused by two mechanisms: the shunt effect through diffuse alveolar damage and the dead space effect through thrombotic injuries in microvascular beds [7-9] (Fig. 2). Rapid multiplication and release of
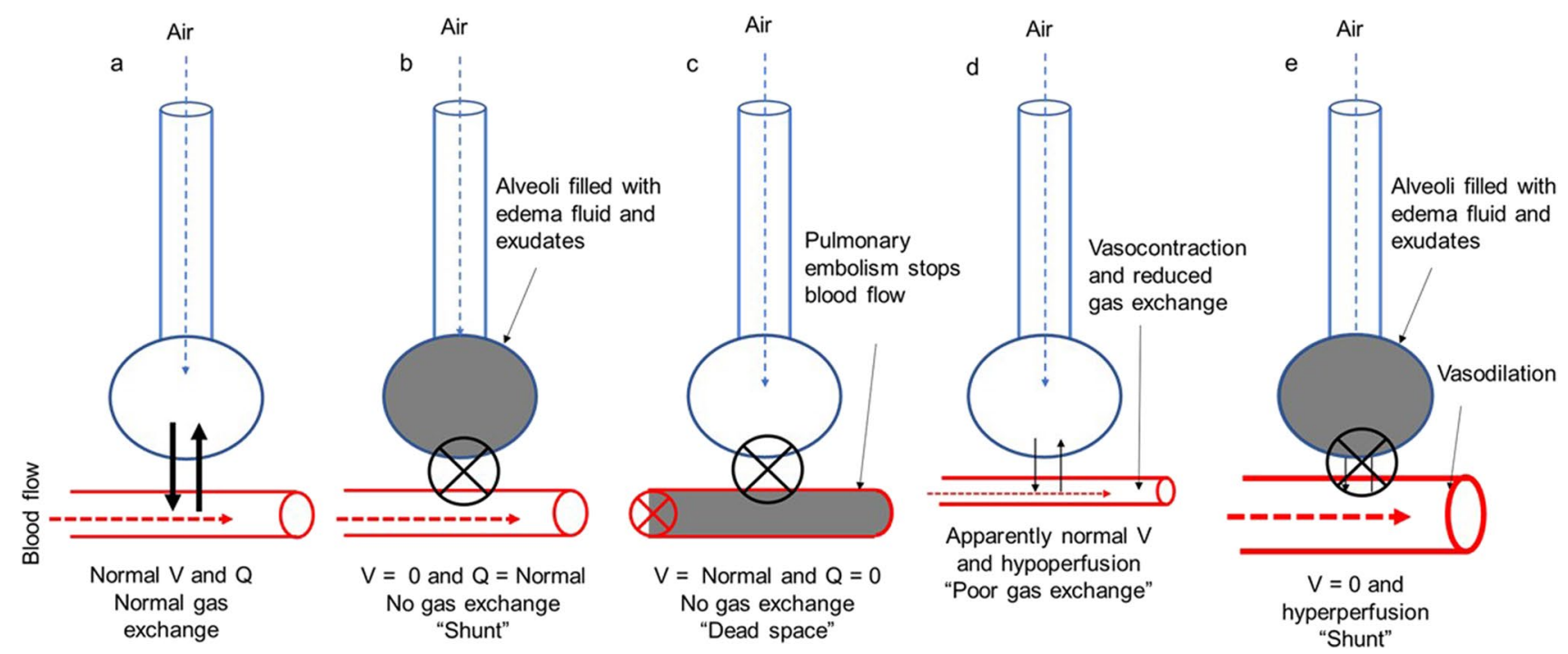

Fig. 1 Normal ventilation and perfusion are responsible for gas exchange in alveoli (a). Alveolar sac filled with edema and exudates produces hypoxemia by decreasing the alveolar and arterial oxygen level result in shunt (b). Pulmonary embolism develops high ventilation in proportion to perfusion produce a dead space (c). Hypoperfusion to the apparently normal parenchyma due to vasoconstriction of pulmonary blood vessels results in poor gas exchange (d) and hyperperfusion through dilated capillaries to alveolar sac filled with edema and exudates result in shunt (e) 
Fig. 2 Normal lung (left) showing smooth blood flow and the effective gas exchange is recognized as normal ventilation and perfusion. COVID-19 infection causes an intense inflammatory reaction (right) results in shunt or dead space or both. Uncontrolled activation of lymphocytes, neutrophil, and pulmonary production of platelets cause lung tissue damages. The virulence in COVID-19 triggers pulmonary microthrombi, endothelial damage, and vascular leakage. The host intends to control the thrombi formation by vigorous fibrinolysis, and degraded fibrin (D-dimer) are released in blood stream. Image Source-https:// doi.org/10.1111/jth.14975

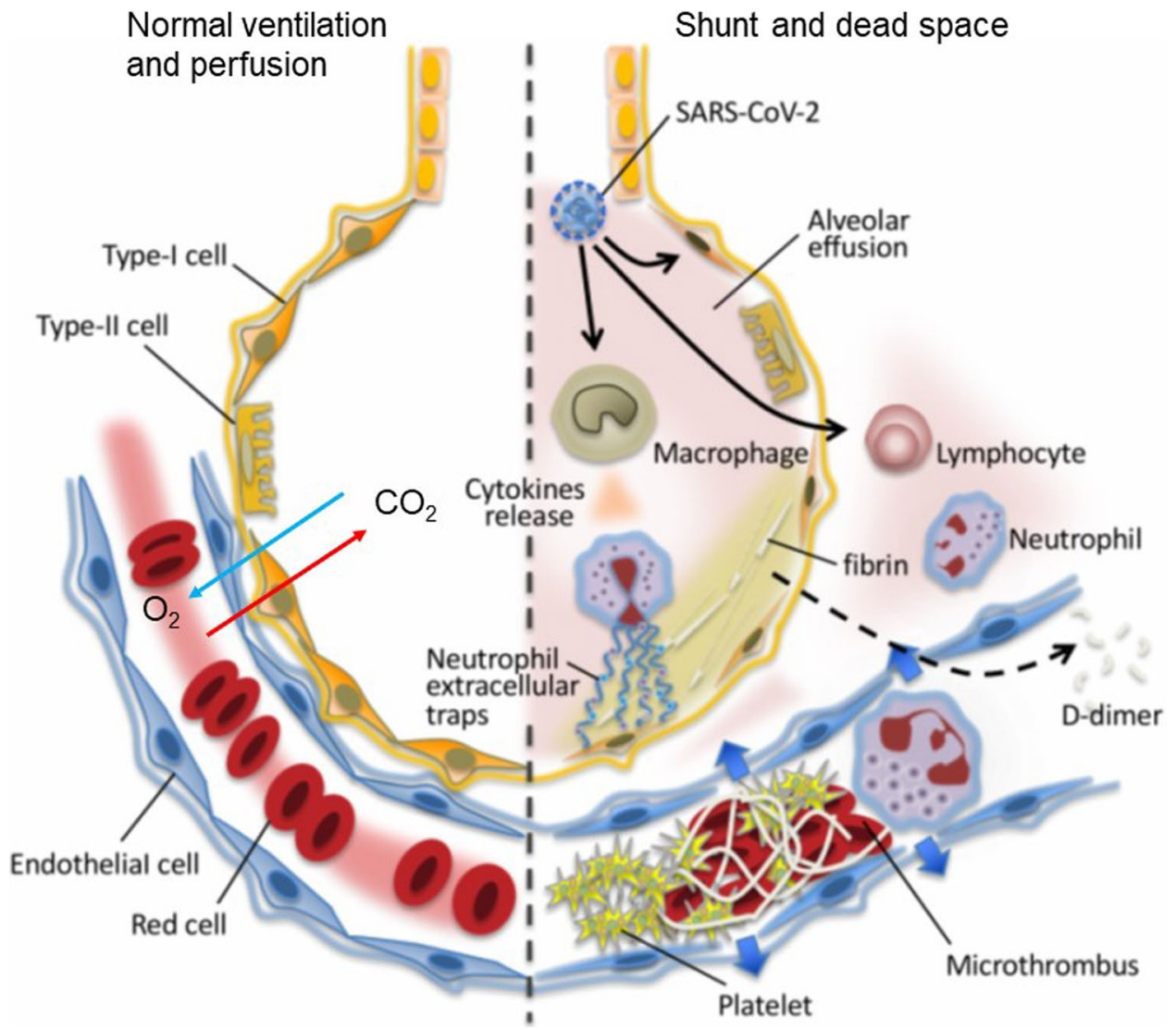

\section{Imaging Modalities}

endothelial cells. It results in vascular leakage and alveolar edema [10]. The accumulation of fluid in the damaged lung parenchyma results in a shunt. The damaged parenchyma appears as GGO, consolidation, septal thickening on conventional chest $\mathrm{CT}$ scans, and perfusion abnormalities on contrast-enhanced CT [11]. COVID-19 viral infection may accelerate microvascular thrombosis followed by pulmonary embolism results in VQ mismatch and hypoxemias [12].

Although portable chest X-rays and lung ultrasound, valuable imaging tools provide limited granular details about lung parenchyma, they are unable to assess pulmonary vasculature/blood flow. Advanced imaging modalities such as high-resolution CT (HRCT), ultra-HRCT (U-HRCT), dual-energy CT (DECT), CT pulmonary angiogram (CTPA), single-photon emission tomography (SPECT)-CT, and electrical impedance tomography (EIT) are useful imaging tools to visualize ventilation-perfusion abnormalities that lead to a VQ mismatch in COVID-19 and are described in the following sections. While EIT does not involve radiation exposure, other imaging modalities involved either administration of radioactivity or exposure to X-rays and require scanning suits. A summary of these imaging modalities to assess lung manifestation in COVID-19 is presented in Table 1.

\section{High-Resolution CT (HRCT) and Ultra-HRCT (U-HRCT)}

Functional respiratory imaging (FRI) technique from Fluidda, Inc. (https://www.fluidda.com/) when combined with HRCT or U-HRCT scans could provide an assessment of pulmonary air tracks and blood volume distribution in the pulmonary blood vessels. The technique requires inspiratory and expiratory scans but does not require a contrast agent. FRI works optimally when the CT slice thickness is $<1 \mathrm{~mm}$. HRCT (slice thickness: 0.625-1.25 mm) and U-HRCT (slice thickness: $0.2-0.5 \mathrm{~mm}$ ) in which thin-slice chest images are acquired and reconstructed using a sharp algorithm are outstanding imaging tools to access blood volume and vasculature abnormalities in COVID-19 without contrast agents. In some longitudinal studies, HRCT detects early lung injury and evaluates disease severity, clinical typing, type of lesions, and post-treatment follow-up [13-17]. U-HRCT scanner observed superiority over HRCT in detecting not only distribution and lesions of COVID-19 but also imaging local lung volume loss $[18,19]$.

Some COVID-19 patients have normal lung compliance and pulmonary blood volume but significant hypoxemia. 


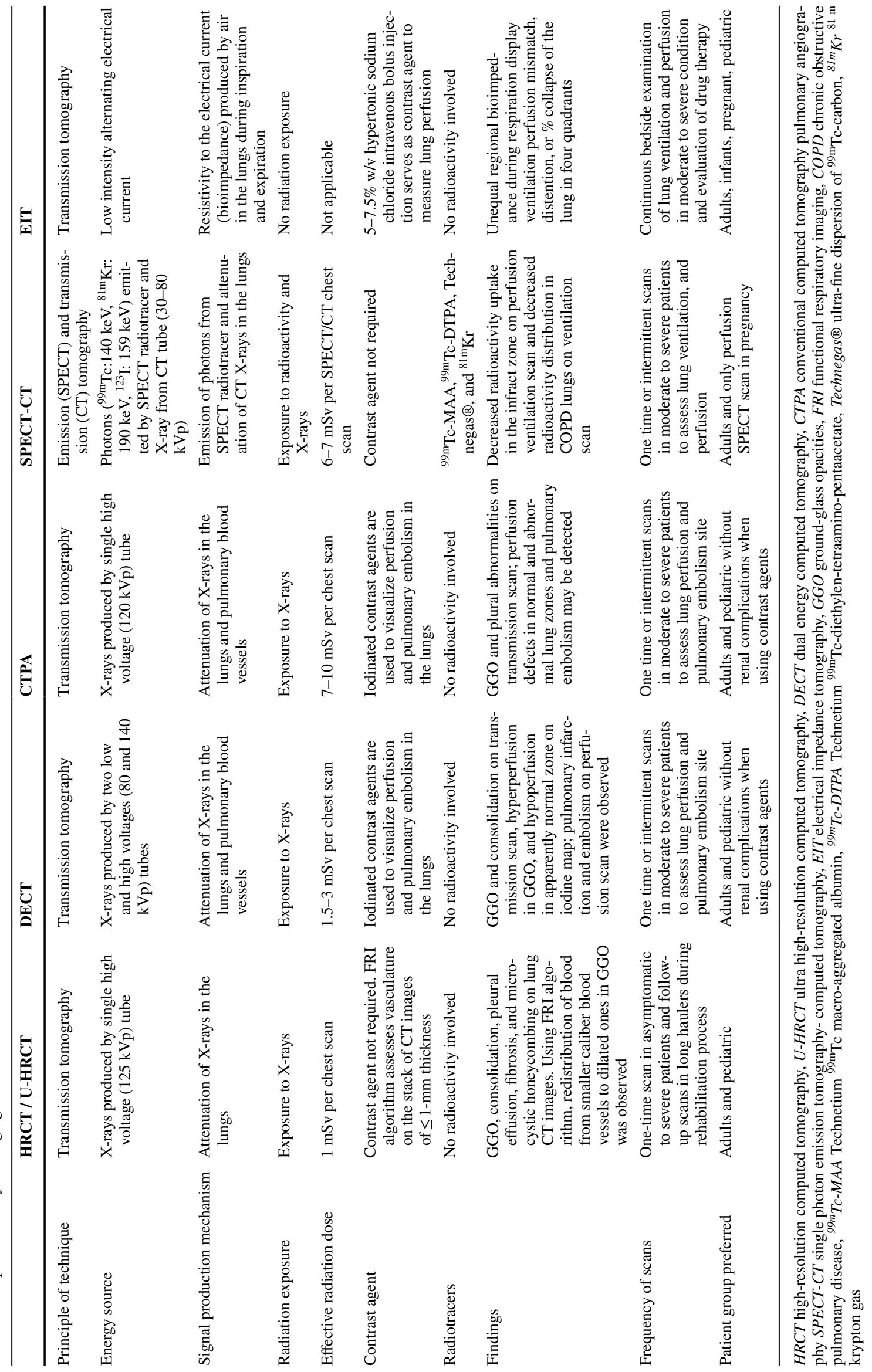


FRI combined with blood vessel (BV) segmentation algorithm on HRCT scans found that patients with COVID-19 had significantly reduced blood volume in the smaller caliber blood vessels (BV5, cross-sectional area $<5 \mathrm{~mm}^{2}$ ) compared with matched acute respiratory distress syndrome (ARDS) patients without COVID-19 and healthy controls [20, 21]. In the long hauler COVID-19 patients, the blood vessels remain anomalous for a long time. The mid-size vessels (BV5_10, cross-sectional area of $5-10 \mathrm{~mm}^{2}$ ) seem to remain dilated which could indicate sustained microvascular obstruction and endothelial damage. In acute COVID-19, they had a significantly higher proportion of blood volume within large-caliber vessels (BV10, cross-sectional area of $>10 \mathrm{~mm}^{2}$ ) (Fig. 3). Impaired gas exchange in the lungs may be due to redistribution of blood away from the small-caliber pulmonary vessels. This mechanism could be due to increased vascular resistance caused by either vasoconstriction of distal pulmonary arteries or the presence of microthrombi or both. The vasculopathy in the lungs reduces gas diffusion capacity causing hypoxemia. FRI technique reaffirms that high levels of positive end-expiratory pressure (PEEP) may not alone be enough to inflate alveoli and facilitate gas exchange, but anticoagulant or pulmonary vasodilators therapies should assist to reduce hypoxemia in these patients [20,21]. A major challenge in the pulmonary rehabilitation process of COVID-19 long-haulers is persisting symptoms long after recovery from infection. The persistent symptoms often include brain fog, fatigue, headaches, dizziness, and shortness of breath [22]. Tracking pulmonary structural and functional information during treatment of COVID-19 long-haulers, FRI possibly could evaluate the development of an optimal therapeutic solution.

\section{Dual-Energy CT (DECT) and CT Pulmonary Angiogram (CTPA)}

Both DECT and CTPA are used to diagnose pulmonary perfusion and embolism in COVID-19 patients. Intravenous administration of iodine-containing contrast agent image pulmonary arteries. Compared with CTPA, DECT allows a significant $50 \%$ reduction in iodine dose while improving signal intensity, maintaining signal-to-noise ratio with a comparable radiation dose [23]. DECT utilizes high- and low-kilovoltage series X-ray energy spectra during image acquisitions and detects perfusion abnormalities. The technique reduces radiation exposure, increases the attenuation of blood vessels at lower energies, and simulates true unenhanced images [11, 24-27].

In the early phase, pulmonary DECT angiography revealed a hyperperfused dilated capillary system in GGO

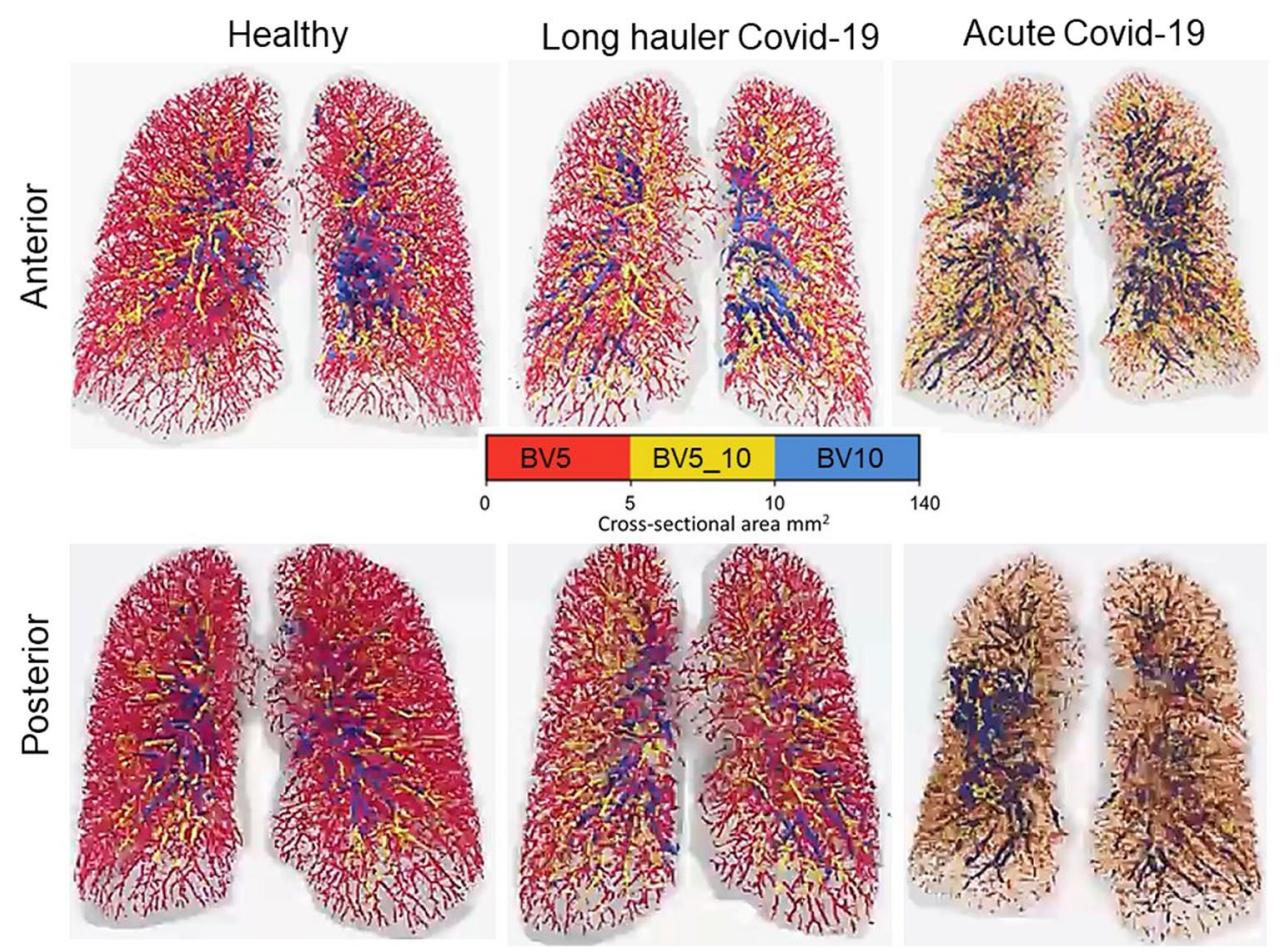

Fig. 3 Functional respiratory imaging on HRCT scans found that the COVID-19 patients had significantly reduced blood volume in the smaller caliber blood vessels (BV5, cross-sectional area $<5 \mathrm{~mm} 2$ ) compared with the long hauler COVID-19 subjects and healthy controls. In the long haulers, the blood vessels remain anomalous for a long time. The mid-size vessels, indicated in yellow (BV5_10, cross-sectional area of 5-10 $\mathrm{mm}^{2}$ ), seem to remain dilated which could indicate sustained microvascular obstruction and endothelial damage. In acute COVID-19, they had a significantly higher proportion of blood volume within large-caliber vessels (BV10, cross-sectional area of $>10 \mathrm{~mm}^{2}$ ) and mainly projected towards the posterior part of the lungs. Impaired gas exchange in the lungs seen in COVID-19 may be partially a result of redistribution of blood away from the small-caliber pulmonary vessels. Image Source-Fluidda Inc 
A
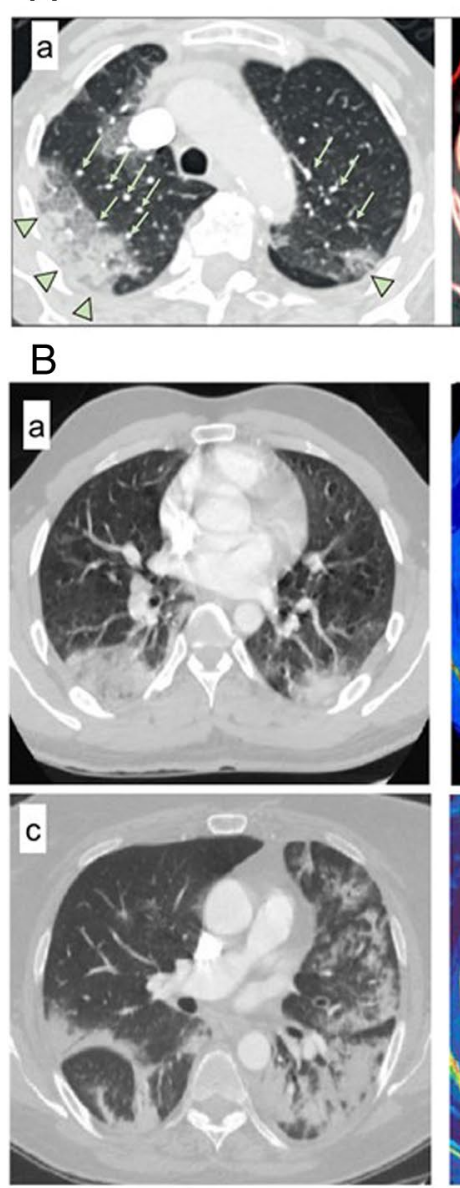
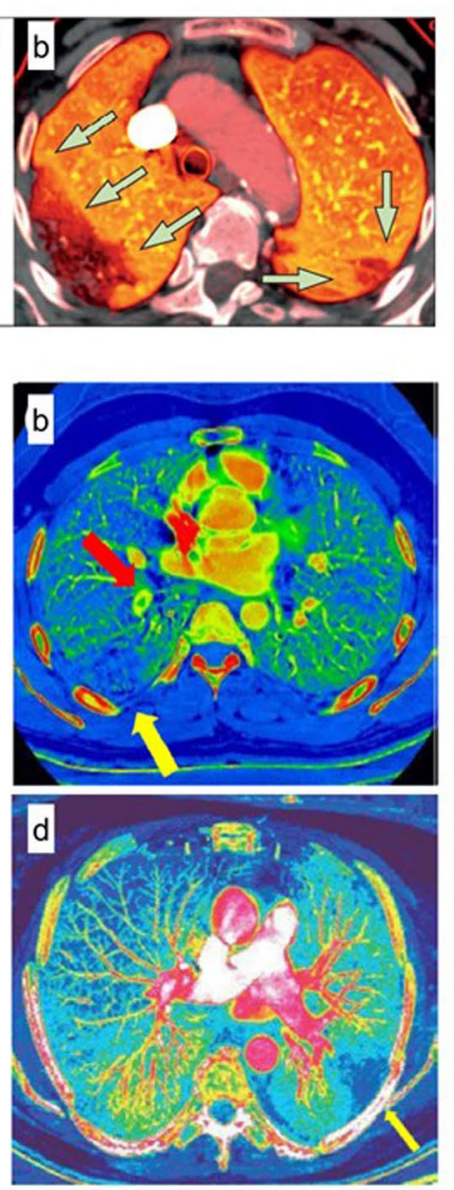

C
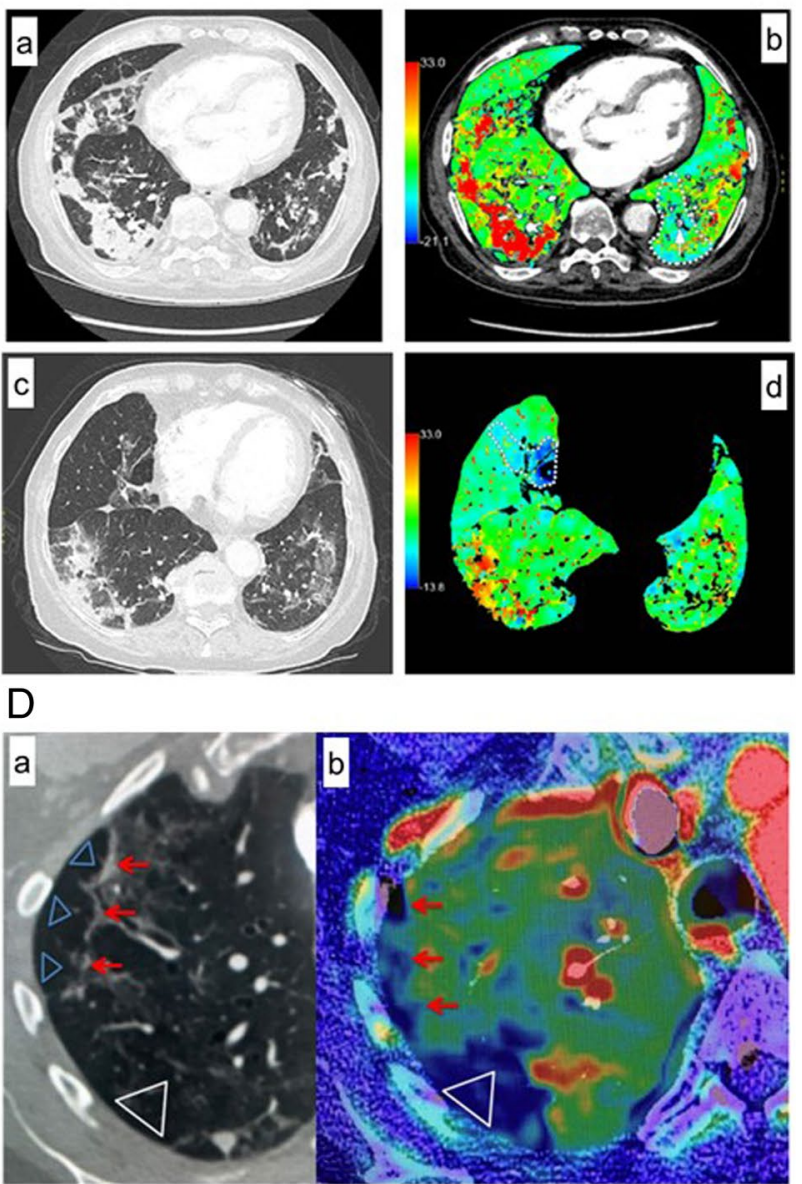

Fig. 4 Panels display perfusion CT scans with dual energy CT (4A, 4B, and 4C) and conventional CT (4D) in COVID-19 patients. In panel 4A, (a) Peripheral GGO and consolidation within the right upper lobe and smaller GGO in the posterior left upper lobe (green arrowheads) are accompanied by dilated subsegmental vessels proximal to, and within, the opacities (green arrows). (b) The accompanying pulmonary blood volume image shows higher perfusion (green arrows) in GGO and consolidation. Image source-https://doi.org/ 10.1016/S1473-3099(20)30367-4. In panel 4B, (a, c) axial CT image shows wedge-shaped bilateral opacities with surrounding GGO; (b) iodine map image shows a triangular peripheral area of decreased perfusion (yellow arrow) in the right lower, distal to pulmonary embolism (red arrow) lobe compatible with pulmonary infarction; (d) iodine map images showing a peripheral, triangular, and hypoperfused area in the left lower lobe (yellow arrow) suggestive of pulmonary infarction. Image source-https://doi.org/10.1016/j.rec. 2020.04.013. In panel 4C, (a) axial CT image shows central GGO and peripheral consolidation a right inferior lobe. (b) Axial iodine color map shows high iodine concentrations in consolidations, and hypoperfusion in left medio-basal segment (dotted line), secondary to the thrombosis of corresponding segmental pulmonary artery (white arrow). (c) Axial CT image shows consolidation in a right inferior lobe and GGO in both inferior lobes. (d) Axial iodine color map shows hypoperfused area in the middle lobe (dotted line). Right inferior lobe consolidation shows high and heterogeneous iodine levels. Image source-https://doi.org/10.21037/qims-20-708. In panel 4D, (a) Axial chest CT of the right upper lobe with subpleural pneumonia (red arrows), surrounded by small GGOs. (b) CTPA shows multiple small subpleural perfusion defects (red arrows) and a larger perfusion defect dorsal in the normal ventilated right upper lobe $(\Delta \mathrm{a})$, due to microvascular obstruction $(\Delta \mathrm{b})$. Pulmonary emboli in the right pulmonary upper lobe were not observed. Image sourcehttps://doi.org/10.1259/bjr.20200718

in COVID-19 patients. Enlarged vessels entering GGO are suggested to occur due to hyperemia and vasodilation through activation of bradykinin receptors. Increased blood flow in poorly ventilated areas results in shunt (Fig. 4A,C) $[11,25]$. In a prolonged disease course, GGO becomes denser (consolidation). The hypoperfusion in consolidated areas suggests obstruction or blockage of capillaries due to thrombus formation. The consolidation was found encircled by hyperperfused blood vessels (Fig. 4B) [24, 27]. During the long hospitalization of the patients, DECT evaluates the possible onset of pulmonary infarcts secondary to pulmonary thromboembolism. In this clinical scenario, dual-energy pulmonary angiography allows differentiation between GGO and ischemic or infarcted areas (Fig. 4B) [19]. Pulmonary perfusion evaluated by iodine concentration maps shows extreme heterogeneity in COVID-19 patients and lower iodine levels in normal parenchyma. DECT revealed a significant number of pulmonary ischemic 
areas although thrombosis remained undetected on images (Fig. 4C) [25].

CTPA detected impaired pulmonary ventilation, pulmonary embolism, and hypoperfusion areas in a COVID-19 patient with elevated D-dimer level [28], and revealed multiple pulmonary perfusion defects without demonstrable pulmonary embolism even before D-dimer elevation (Fig. 4D) [29].

\section{SPECT-CT}

VQ scintigraphy was recommended to detect pulmonary embolism and ventilation-perfusion mismatch in COVID19 [30-33], where CT confronts limitations. Perfusion scans are performed using hybrid SPECT-CT cameras with intravenous injection of Technetium ${ }^{99 \mathrm{~m}} \mathrm{Tc}$ macroaggregated albumin ( ${ }^{99 \mathrm{~m}}$ Tc-MAA) and ventilation scans with inhalation of ${ }^{81 \mathrm{~m}} \mathrm{Kr}$-Krypton gas or Technetium
${ }^{99 \mathrm{~m}} \mathrm{Tc}$-diethylen-tetraamino-pentaacetate ( $\left.{ }^{99 \mathrm{~m}} \mathrm{Tc}-\mathrm{DTPA}\right)$ or ultra-fine dispersion of ${ }^{99 \mathrm{~m}} \mathrm{Tc}-$ Carbon (Technegas $\left.{ }^{\circledR}\right)$ [34]. The biodistribution of radiotracers in the lungs is then measured to observe ventilation and perfusion defects. Decreased radiotracer uptake and no mismatch of $\mathrm{V}$ and Q SPECT scans are indicative of a low possibility of pulmonary embolism. The regions with decreased radiotracer uptake are examined for peripheral parenchymal GGO on CT scans of COVID-19 patients (Fig. 5A) [31]. Ventilation SPECT-CT scan revealed increased Technegas $\AA$ uptake in the tracheobronchial tract in COVID-19 patients suggesting blockage of airway paths (bronchus, bronchi, and bronchioles) in the lungs and cautioning signs of a tracheobronchitis. In the absence of previous pulmonary disease, ventilation SPECT-CT might have diagnostic and therapeutic applications (Fig. 5B) [30]. In the early phase, COVID-19 patients have shown decreased ventilation and normal perfusion in the lung regions where GGO or consolidation was observed on CT scans (Fig. 5C) [31].
A

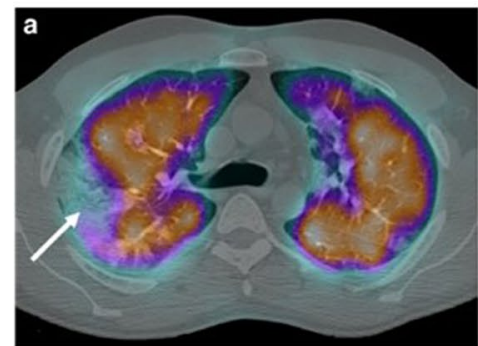

b

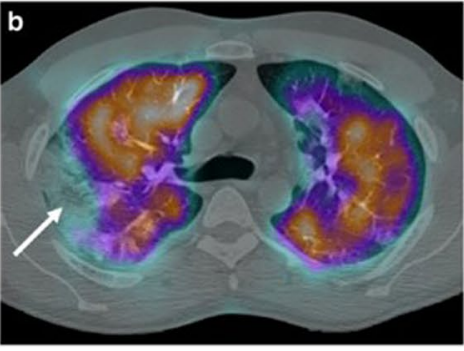

C

a

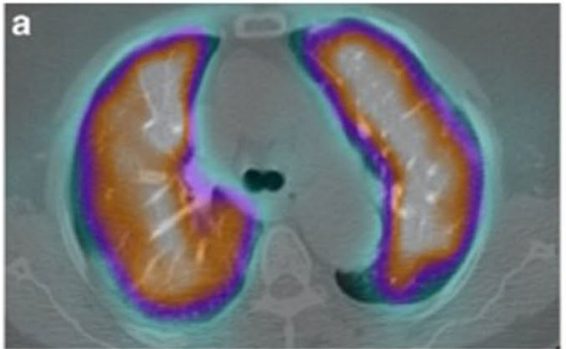

d
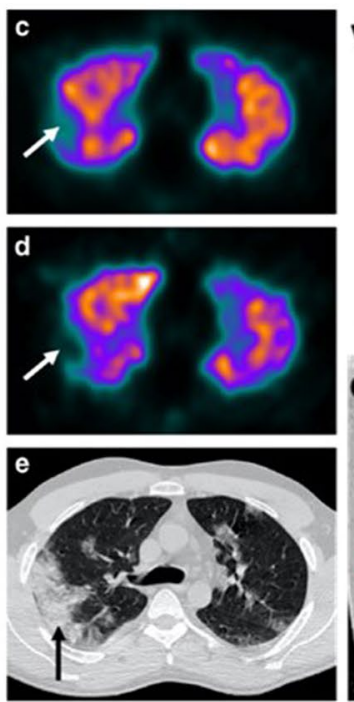

B

Ventilation
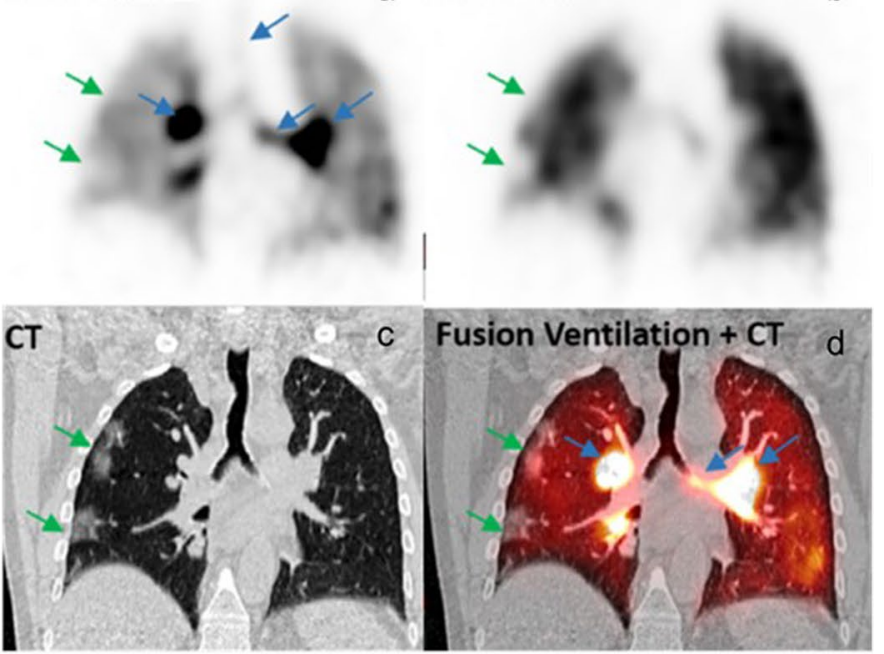

a

Perfusion
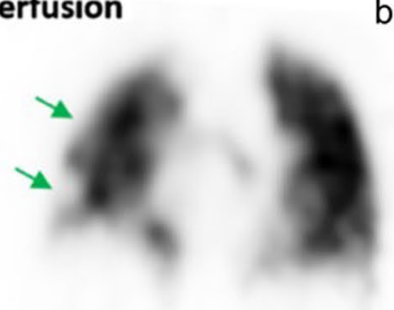

b

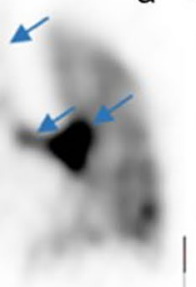

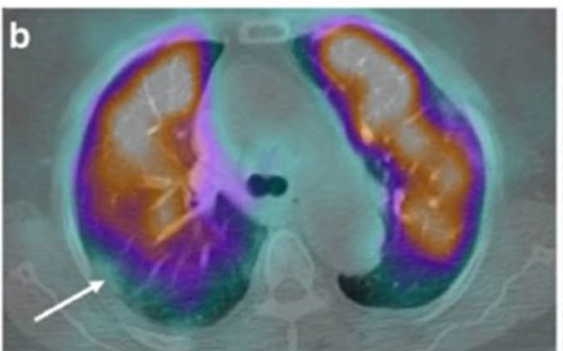

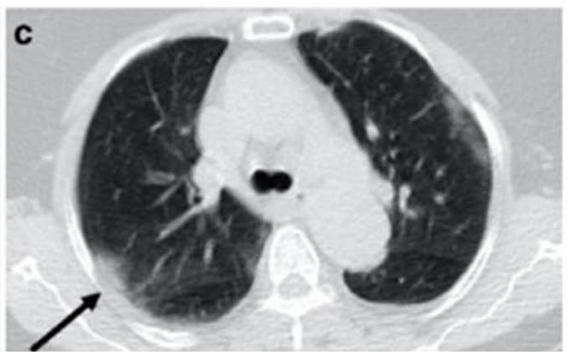

Fig. 5 In panel 5A, the axial images showing decreased radiotracer uptake in both perfusion and ventilation scan (white arrow) in fused SPECT-CT $(\mathbf{a}, \mathbf{b})$ indicating low probability of pulmonary embolism. SPECT $(\mathbf{c}, \mathbf{d})$ observed defect matches with the posterior segment of the right upper lobe alveolar filling (black arrow) on the CT scan (e). In panel 5B, on coronal images, decreased dual radiotracer uptake (green arrows) in ventilation (a) and perfusion (b) SPECT scans indicates low possibility of pulmonary embolism. Increased tracheobronchial tract uptake of (blue arrows), with marked intensity on the proximal bronchi suggesting tracheobronchitis or chronic obstructive pulmonary disease. In panel 5C, the axial SPECT images showing normal perfusion scan (a) and defects in ventilation scan (b) in the posterior segment of the right upper lobe (white arrow) that matches with the GGO on CT scan (c, black arrow). Image source-https://doi.org/10.1007/s00259-020-04920-w, https://doi.org/10.1007/s00259-020-04834-7 
Fig. 6 In this study, EIT showed real-time noninvasive bedside ventilation and perfusion in hypoxemic COVID-19 patients with respiratory failure. All the three COVID-19 patients intubated for acute hypoxic respiratory failure $\left(\mathrm{PaO}_{2} / \mathrm{FiO}_{2}<300\right)$ had different respiratory system compliance. EIT was used to determine regional ventilation and perfusion distribution. On CT scans, case 1 shows peripheral and basilar GGO, while case 2 and 3 describe diffuse bilateral GGO. In case 1, EIT showed severe right-lung perfusion anomalies, homogenous ventilation, and a moderate decrease in respiratory compliance $\left(40 \mathrm{ml} / \mathrm{cm} \mathrm{H}_{2} \mathrm{O}\right)$. Case 2 and case 3 showed a progressive decrease of respiratory compliance, without major perfusion disturbances. $\mathrm{PaO}_{2} / \mathrm{FiO}_{2}$ means ratio of arterial oxygen partial pressure in $\mathrm{mmHg}$ to fractional inspired oxygen expressed as fraction. Image source-https:// doi.org/10.1164/rccm.2020051801IM
Case 1
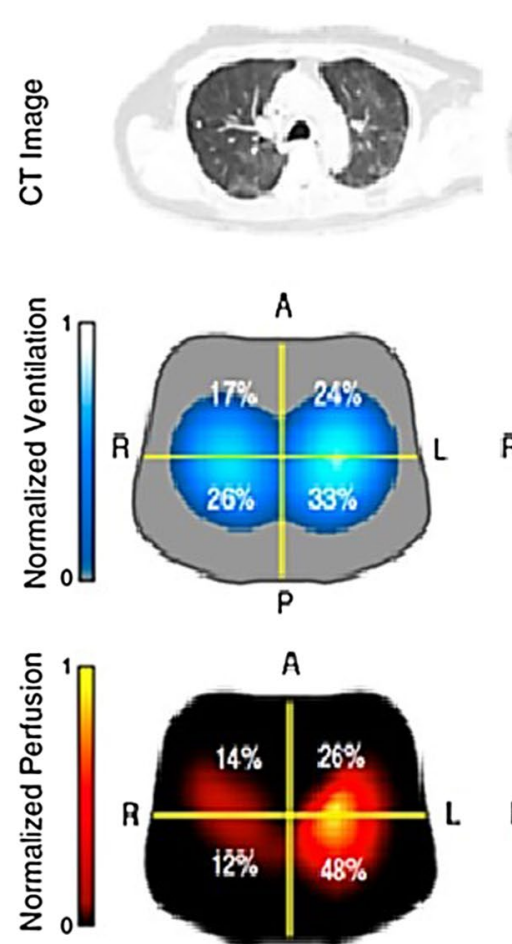

A

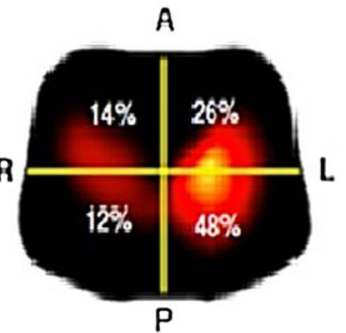

$\mathrm{PaO}_{2} / \mathrm{FiO}_{2}$ :

205

$\mathrm{C}_{\mathrm{RS}}\left(\mathrm{mU}_{\mathrm{CmH}} \mathrm{O}\right):$

Case 2

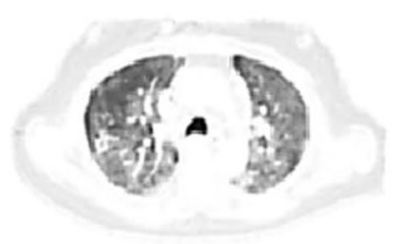

A

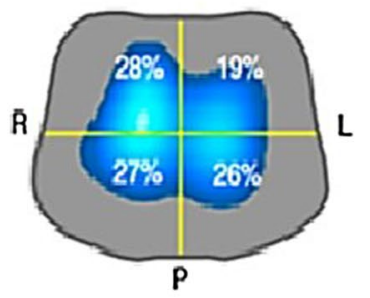

A

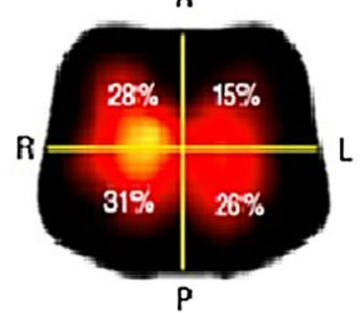

170

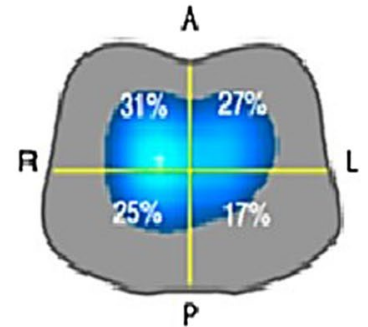

A.

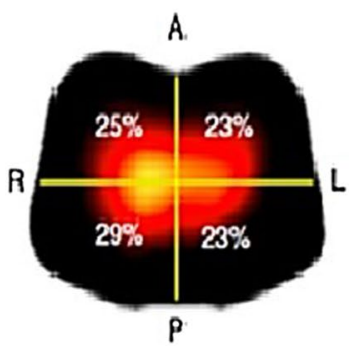

195

11
Ventilation SPECT scans are recommended if CTPA is inconclusive, contraindicated, and in exceptional circumstances in COVID-19 confirmed or suspected cases [35, 36]. VQ SPECT-CT not only performs diagnosis of pulmonary embolism but also assesses the degree of pulmonary involvement avoiding the risk of renal failure linked to the injection of iodinated contrast agents to the patients at risk [37]. Perfusion SPECT-CT avoids unnecessary risk related to aerosolization of respiratory droplets and possible viral spreada risk factor associated with ventilation SPECT scan. It is therefore recommended as a choice for detecting pulmonary embolism in COVID-19 patients [38, 39]. In pregnancy, perfusion SPECT without $\mathrm{CT}$ reduces exposure to radiation dose. However, in certain cases, if the pregnant woman falls in the clinical "vulnerable group" for COVID-19 infection, a perfusion SPECT with low-dose CT is advocated [34, 35].

\section{Electrical Impedance Tomography (EIT)}

Imaging tools like EIT can monitor lung ventilation and perfusion bedside while the patient is receiving treatment. It is a non-invasive radiation-free functional imaging modality that allows continuous bedside monitoring of lung ventilation and perfusion in real time [40]. EIT uses electric current and evaluates the distribution of alternating current conductivity within the thoracic cavity. It is based on the repeated measurement of the surface voltages $(40-50$ cycles/s) resulting from a rotating high frequency $(50-80 \mathrm{kHz})$ and low intensity $(5-10 \mathrm{~mA})$ alternating current that circulates between the electrodes around the thorax. The spatial resolution can be improved with the increasing number of electrodes. With a computer and electrodes applied to the patient's chest, the bioimpedance (resistance) signal can be processed allowing the description of tissue characteristics (conductivity) located in the selected body circumference. The electrodes collect information on impedance by forming a relative image based on a reference. While the tissues allow the passage of current with little resistance, the air in the lungs offers high resistance [41]. The impedance of the lung during inspiration is double that of expiration. EIT allows the estimation of the tidal volume, the percentages of the tidal volume distribution in each quadrant or layer, the minimum impedance (end-expiratory lung impedance), or maximum impedance (total lung capacity) [40]. The quantification of pulmonary perfusion by EIT can be performed by a brief breath-hold, followed by rapid infusion of hypertonic saline $(10 \mathrm{ml}, 5-7.5 \% \mathrm{w} / \mathrm{v}$ $\mathrm{NaCl}$ ), which dramatically reduces chest impedance, thus acting as an intravascular contrast [40]. In the pigs, pulmonary 
perfusion maps by EIT were found to well agree with perfusion maps produced using SPECT [42] and positron emission tomography [43].

EIT was found helpful to characterize the etiology of hypoxemia of COVID-19 patients with respiratory failure (Fig. 6) [44]. COVID-19 pneumonia requires different ventilator strategies. For patients who have VQ mismatch, EIT could assist in identifying different pneumonia patterns and setting optimal parameters on a mechanical ventilator [45]. COVID-19 patients often have airway secretions that severely compromise ventilation. Therapeutic bronchoalveolar lavage removes mucus in the small airways and improves regional ventilation. EIT was found useful at the bedside to monitor the ventilation treatment and anticoagulant treatment in COVID-19 [14, 46].

The procedure to titrate PEEP in COVID-19 or associated ARDS patients is unknown. The survival of patients is improved if higher PEEP successfully recruited partly or completely collapsed lung tissue [47]. However, excessive PEEP caused alveolar overdistention, resulting in reduced patient survival [48]. Therefore, PEEP should be personalized to maximize alveolar recruitment and minimize the amount of alveolar overdistention. In a case series of COVID-19 patients with moderate to severe ARDS, EIT successfully provided a reliable bedside approach to detect both alveolar overdistention and alveolar collapse $[49,50]$.

\section{Concluding Remarks}

COVID-19 hypoxemic patients exhibit abnormal hyperperfusion in areas of lung GGO and show abnormally decreased perfusion in areas of the apparently normal lung parenchyma. Hypoperfusion of apparently healthy areas could be a consequence of vasoconstriction due to the accumulation of angiotensin II that shifts vascular flow towards areas of nonaerated hyperperfused lung in moderate to severe COVID-19 cases. In most COVID cases, the posterior part of the lungs is severely affected. Prone sleeping position shifts blood flow through gravity effect towards hypoperfused anterior part of the apparently normal lungs and improves oxygenation. The severity of the disease and degree of VQ mismatch in COVID patients makes them different from one another clinically.

FRI on HRCT revealed that the total pulmonary blood volumes as measured do not vary significantly between healthy and COVID-19 patients [21]. Throughout the pulmonary vasculature, the blood flow was redistributed from small vessels to medium and larger blood vessels entering in GGO and consolidation. The measurements of blood volume distribution, anatomical changes of pulmonary vasculature may offer a powerful means to evaluate effective treatments in acute COVID-19 patients or recovering long haulers [20,21]. FRI findings correlate with the DECT and CTPA iodine perfusion maps where high iodine concentration was observed in GGO and lower iodine levels in normal parenchyma with or without evidence of thrombosis or pulmonary embolism [11, 25].

DECT allows a significant reduction in iodine dose while improving the intravascular signal intensity and maintaining a signal-to-noise ratio with comparable radiation dose. Although the pulmonary vasculature and embolism can be seen in exquisite detail with CTPA and DECT, they are reserved for symptomatic patients with specific clinical indications [51]. VQ SPECT scan with low-dose CT identifies the dual pathology of pulmonary embolism and COVID-19 related lung parenchymal abnormalities. In pregnant patients with suspected massive pulmonary embolism, only perfusion scintigraphy is recommended. A low-dose CT with SPECT perfusion scan increases sensitivity to $~ 97 \%$, compared with CTPA, and a significant increase in specificity (97.2-100\%) to detect pulmonary embolism in COVID patients [35]. The absorbed fetal dose from the low-dose CT is negligible $(<0.1 \mathrm{mSv})$, while radiation exposure reduces 5 times compared with $9 \mathrm{mSv}$ dose received from CTPA [52, 53]. Allergy to iodinated contrast agents and renal failure in COVID patients restricts the use of iodinated contrast agents on the CT scan [54]. In this case, pulmonary scintigraphy is recommended for diagnosing pulmonary embolism in COVID-19. The radiation exposure and the need for decontaminated scanning room limit the serviceability of SPECT or CT for routine use. In this situation, EIT is emerging as a promising clinical imaging tool for continuous and real-time monitoring of pulmonary ventilation and perfusion in COVID-19 patients. The complete discussion of the specific pros and cons associated with the different imaging modalities is outside the scope of this review. We emphasize the clinical usefulness of imaging in the settings of COVID-19.

Acknowledgements The authors gratefully acknowledge University of Southern California; The Saban Research Institute for providing literature survey facilities and Fluidda Inc. for useful input in the group discussion.

\section{Declarations}

Ethics Approval This article does not contain any studies with human participants or animals performed by any of the authors.

Conflict of Interest The authors declare that they have no conflict of interest.

\section{References}

1. Hemmings HC, Egan TD (2019) Pharmacology and physiology for anesthesia: foundations and clinical application. Second edition. ed., Philadelphia, PA: Elsevier, Inc.

2. Lumb AB, Horner D (2013) Chapter 25-Pulmonary physiology. In Pharmacology and Physiology for anesthesia, Eds. Hemmings HC, Egan TD. Philadelphia: W.B. Saunders, pp 445-457.

3. Levitzky MG (2018) Pulmonary physiology. Ninth edition. ed., New York: McGraw-Hill Education.

4. Nitsure M, Sarangi B, Shankar GH et al (2020) Mechanisms of hypoxia in COVID-19 patients: a pathophysiologic reflection. Indian J Crit Care Med 24:967-970

5. Sarkar M, Niranjan N, Banyal PK (2017) Mechanisms of hypoxemia Lung India 34:47-60 
6. Petersson J, Glenny RW (2014) Gas exchange and ventilation-perfusion relationships in the lung. Eur Respir J 44:1023-1041

7. Abourida Y, Rebahi H, Chichou H et al (2020) What open-lung biopsy teaches us about ARDS in COVID-19 patients: mechanisms, pathology, and therapeutic implications. Biomed Res Int 2020:2909673

8. Hanley B, Naresh KN, Roufosse C et al (2020) Histopathological findings and viral tropism in UK patients with severe fatal COVID19: a post-mortem study. Lancet Microbe 1:e245-e253

9. Iba T, Levy JH, Levi M, Thachil J (2020) Coagulopathy in COVID19. J Thromb Haemost 18:2103-2109

10. Yap JKY, Moriyama M, Iwasaki A (2020) Inflammasomes and pyroptosis as therapeutic targets for COVID-19. J Immunol 205:307-312

11. Lang M, Som A, Mendoza DP et al (2020) Hypoxaemia related to COVID-19: vascular and perfusion abnormalities on dual-energy CT. Lancet Infect Dis 20:1365-1366

12. Dhawan RT, Gopalan D, Howard L et al (2021) Beyond the clot: perfusion imaging of the pulmonary vasculature after COVID-19. Lancet Respir Med 9:107-116

13. Lu X, Gong W, Peng Z, Zeng F, Liu F (2020) High resolution CT imaging dynamic follow-up study of novel coronavirus pneumonia. Front Med (Lausanne) 7:168

14. Fu Y, Zou R, Wang S et al (2020) Monitoring bronchoalveolar lavage with electrical impedance tomography: first experience in a patient with COVID-19. Physiol Meas 41:085008

15. Mazzolini M, Monari M, Angeletti G, Dalpiaz G, Rocca A (2021) Fatal pulmonary fibrosis complicating COVID-19 infection in preexistent emphysema. Radiol Case Rep 16:361-363

16. Majidi H, Bani-Mostafavi ES, Mardanshahi Z et al (2020) High-resolution computed tomography finding in 552 patients with symptomatic COVID-19: first report from north of Iran. Emerg Radiol 27:633-639

17. Khaliq M, Raja R, Khan N, Hanif H (2020) An analysis of high-resolution computed tomography chest manifestations of COVID-19 patients in Pakistan. Cureus 12:e9373

18. Kakinuma R, Moriyama N, Muramatsu Y et al (2015) Ultra-high-resolution computed tomography of the lung: image quality of a prototype scanner. PLoS One 10:e0137165

19. Iwasawa T, Sato M, Yamaya T et al (2020) Ultra-high-resolution computed tomography can demonstrate alveolar collapse in novel coronavirus (COVID-19) pneumonia. Jpn J Radiol 38:394-398

20. Thillai M, Patvardhan C, Swietlik EM, et al. (2020) Functional respiratory imaging identifies redistribution of pulmonary blood flow in patients with COVID-19. Thorax.

21. Lins M, Vandevenne J, Thillai M et al (2020) Assessment of small pulmonary blood vessels in COVID-19 patients using HRCT. Acad Radiol 27:1449-1455

22. Greenhalgh T, Knight M, A'Court C, Buxton M, Husain L (2020) Management of post-acute covid-19 in primary care. BMJ 370:m3026

23. Yuan R, Shuman WP, Earls JP et al (2012) Reduced iodine load at CT pulmonary angiography with dual-energy monochromatic imaging: comparison with standard CT pulmonary angiography - a prospective randomized trial. Radiology 262:290-297

24. Marsico S, Espallargas Gimenez I, Carbullanca Toledo SJ, Del Carpio Bellido LA, Maiques Llacer JM, Zuccarino F (2020) Pulmonary infarction secondary to pulmonary thromboembolism in COVID-19 diagnosed with dual-energy CT pulmonary angiography. Rev Esp Cardiol (Engl Ed) 73:672-674

25. Grillet F, Busse-Cote A, Calame P, Behr J, Delabrousse E, Aubry S (2020) COVID-19 pneumonia: microvascular disease revealed on pulmonary dual-energy computed tomography angiography. Quant Imaging Med Surg 10:1852-1862

26. Ganti S, Kok SSX, Aftab S, Halagatti SV, Wong SBS, Uppaluri AS (2021) Spectrum of lung perfusion changes on dual-energy CT in COVID-19: incremental benefit to conventional CT. Emerg Radiol.

27. von der Thusen JH, Ghariq E, Overbeek MJ et al (2020) Spectrum of vascular involvement in coronavirus disease 2019 pneumonia-findings on CT perfusion. Crit Care Explor 2:e0266

28. Ierardi AM, Angileri SA, Arrichiello A et al (2020) Pulmonary embolism in COVID-19: ventilation and perfusion computed tomography. IDCases 21:e00805

29. Oudkerk M, Kuijpers D, Oudkerk SF, van Beek EJ (2020) The vascular nature of COVID-19. Br J Radiol 93:20200718

30. Verger A, Bahloul A, Melki S, Karcher G, Imbert L, Marie PY (2020) Tracheobronchitis signs observed on ventilation lung scintigraphy during the course of COVID-19 infection. Eur J Nucl Med Mol Imaging 47:2709-2710

31. Cobes N, Guernou M, Lussato D et al (2020) Ventilation/perfusion SPECT/CT findings in different lung lesions associated with COVID19: a case series. Eur J Nucl Med Mol Imaging 47:2453-2460

32. Das JP, Yeh R, Schoder H (2020) Clinical utility of perfusion (Q)single-photon emission computed tomography (SPECT)/CT for diagnosing pulmonary embolus (PE) in COVID-19 patients with a moderate to high pre-test probability of PE. Eur J Nucl Med Mol Imaging.

33. Sciagra R, Rubino F, Malandrino D, Bernardeschi N, Pignone AM, Berni A (2020) Are disease-related pulmonary perfusion abnormalities detectable in COVID-19 patients? Suspicious findings in a lung perfusion SPECT performed for ruling out classical pulmonary embolism. Eur J Nucl Med Mol Imaging 47:2211-2213

34. Bajc M, Schumichen C, Gruning T et al (2019) EANM guideline for ventilation/perfusion single-photon emission computed tomography (SPECT) for diagnosis of pulmonary embolism and beyond. Eur J Nucl Med Mol Imaging 46:2429-2451

35. Voo S, Dizdarevic S (2020) Single photon emission computed tomography lung perfusion imaging during the COVID-19 pandemic: does nuclear medicine need to reconsider its guidelines? Nucl Med Commun 41:991-993

36. Mahaletchumy T, Muhamad M, Mohammad Kazmin NE, Kori N, Periyasamy P (2021) Pulmonary embolism in an asymptomatic COVID-19 patient detected on ventilation/perfusion SPECT/CT. Clin Nucl Med.

37. Burger IA, Niemann T, Patriki D, Fontana F, Beer JH (2020) Lung perfusion [(99m)Tc]-MAA SPECT/CT to rule out pulmonary embolism in COVID-19 patients with contraindications for iodine contrast. Eur J Nucl Med Mol Imaging 47:2209-2210

38. Lu Y, Macapinlac HA (2020) Perfusion SPECT/CT to diagnose pulmonary embolism during COVID-19 pandemic. Eur J Nucl Med Mol Imaging 47:2064-2065

39. Le Roux PY, Le Gal G, Salaun PY (2020) Lung scintigraphy for pulmonary embolism diagnosis during the COVID-19 pandemic: does the benefit-risk ratio really justify omitting the ventilation study? Eur J Nucl Med Mol Imaging 47:2499-2500

40. Tomicic V, Cornejo R (2019) Lung monitoring with electrical impedance tomography: technical considerations and clinical applications. J Thorac Dis 11:3122-3135

41. Holder D, Institute of Physics (Great Britain) (2005) Electrical impedance tomography: methods, history, and applications. Bristol ; Philadelphia: Institute of Physics Pub.

42. Borges JB, Suarez-Sipmann F, Bohm SH et al (1985) (2012) Regional lung perfusion estimated by electrical impedance tomography in a piglet model of lung collapse. J Appl Physiol 112:225-236

43. Bluth T, Kiss T, Kircher M et al (2019) Measurement of relative lung perfusion with electrical impedance and positron emission tomography: an experimental comparative study in pigs. Br J Anaesth 123:246-254

44. Morais CCA, Safaee Fakhr B, De Santis Santiago RR et al (2021) Bedside electrical impedance tomography unveils respiratory "chimera" in COVID-19. Am J Respir Crit Care Med 203:120-121

45. Tomasino S, Sassanelli R, Marescalco C, Meroi F, Vetrugno L, Bove $\mathrm{T}$ (2020) Electrical impedance tomography and prone position during ventilation in COVID-19 pneumonia: case reports and a brief literature review. Semin Cardiothorac Vasc Anesth 24:287-292

46. Safaee Fakhr B, Araujo Morais CC, De Santis Santiago RR et al (2020) Bedside monitoring of lung perfusion by electrical impedance tomography in the time of COVID-19. Br J Anaesth 125:e434-e436

47. Amato MB, Meade MO, Slutsky AS et al (2015) Driving pressure and survival in the acute respiratory distress syndrome. N Engl J Med 372:747-755

48. Writing Group for the Alveolar Recruitment for Acute Respiratory Distress Syndrome Trial I, Cavalcanti AB, Suzumura EA et al (2017) Effect of lung recruitment and titrated positive end-expiratory pressure (PEEP) vs low PEEP on mortality in patients with acute respiratory distress syndrome: a randomized clinical trial. JAMA 318:1335-1345

49. van der Zee P, Somhorst P, Endeman H, Gommers D (2020) Electrical impedance tomography for positive end-expiratory pressure titration in COVID-19-related acute respiratory distress syndrome. Am J Respir Crit Care Med 202:280-284

50. Frerichs I, Amato MB, van Kaam AH et al (2017) Chest electrical impedance tomography examination, data analysis, terminology, clinical use and recommendations: consensus statement of the translational EIT development study group. Thorax 72:83-93 
51. ACR Recommendations for the use of chest radiography and computed tomography (CT) for suspected COVID-19 infection. https://www.acr. org/Advocacyand-Economics/ACR-Position-Statements/Recommenda tions-for-Chest-Radiography-and-CT-for-Suspected-COVID19-Infec tion.

52. Jewell KE, Lee ST, Trubiano J, Lau E, Scott AM (2020) Ventilation perfusion lung SPECT/CT in pregnancy during COVID-19. Intern Med J 50:1588-1590

53. Khosh SK, Ummmm P, Lee ST, Rowe CC (2014) VQ SPECT/CT Vs CTPA - the impact if changes of breast tissue weighting factor on radiation exposure and cancer risk. Abstracts of the 44th Annual Scientific
Meeting of the Australian and New Zealand Society of Nuclear Medicine (ANZSNM), 25-28 April 2014, Adelaide Australia. Intern Med J 44(Suppl 1):1-38

54. Pan XW, Xu D, Zhang H, Zhou W, Wang LH, Cui XG (2020) Identification of a potential mechanism of acute kidney injury during the COVID-19 outbreak: a study based on single-cell transcriptome analysis. Intensive Care Med 46:1114-1116

Publisher's Note Springer Nature remains neutral with regard to jurisdictional claims in published maps and institutional affiliations. 